\title{
Structure-based design of anticancer prodrug PABA/NO
}

\author{
Xinhua Ji' \\ Ajai $\mathrm{Pal}^{2}$ \\ Ravi Kalathur ${ }^{1}$ \\ Xun $\mathrm{Hu}^{2}$ \\ Yijun $\mathrm{Gu}^{1,2}$ \\ Joseph E Saavedra ${ }^{3}$ \\ Gregory S Buzard ${ }^{3}$ \\ Aloka Srinivasan ${ }^{4}$ \\ Larry K Keefer ${ }^{4}$ \\ Shivendra $V$ Singh ${ }^{2}$ \\ 'Macromolecular Crystallography \\ Laboratory, National Cancer \\ Institute, National Institutes \\ of Health, Frederick, MD 2I702, USA; \\ ${ }^{2}$ Department of Pharmacology \\ and University of Pittsburgh Cancer \\ Institute, University of Pittsburgh \\ School of Medicine, Pittsburgh, \\ PA I52।3, USA; ${ }^{3}$ Basic Research \\ Program, SAIC-Frederick Inc., \\ Frederick, MD 21702, USA; \\ ${ }^{4}$ Laboratory of Comparative \\ Carcinogenesis, National Cancer \\ Institute, National Institutes \\ of Health, Frederick, MD 21702, USA
}

Correspondence: Xinhua Ji $\mathrm{NCl}$-Frederick, 1050 Boyles Street, Frederick, MD 21702, USA

Tel + I $30 \mid 8465035$

Fax + I 3018466073

Email jix@ncifcrf.gov

\begin{abstract}
Glutathione S-transferase (GST) is a superfamily of detoxification enzymes, represented by GST $\alpha$, GST $\mu$, GST $\pi$, etc. GST $\alpha$ is the predominant isoform of GST in human liver, playing important roles for our well being. GST $\pi$ is overexpressed in many forms of cancer, thus presenting an opportunity for selective targeting of cancer cells. Our structure-based design of prodrugs intended to release cytotoxic levels of nitric oxide in GST $\pi$-overexpressing cancer cells yielded PABA/NO, which exhibited anticancer activity both in vitro and in vivo with a potency similar to that of cisplatin. Here, we present the details on structural modification, molecular modeling, and enzymatic characterization for the design of PABA/NO. The design was efficient because it was on the basis of the reaction mechanism and the structures of related GST isozymes at both the ground state and the transition state. The ground-state structures outlined the shape and property of the substrate-binding site in different isozymes, and the structural information at the transition-state indicated distinct conformations of the Meisenheimer complex of prodrugs in the active site of different isozymes, providing guidance for the modifications of the molecular structure of the prodrug molecules. Two key alterations of a GST $\alpha$-selective compound led to the GST $\pi$-selective PABA/NO.
\end{abstract}

Keywords: structure-based, drug design, anticancer, prodrug, PABA/NO

\section{Introduction}

Glutathione $S$-transferases (GSTs, EC 2.5.1.18) are a superfamily of enzymes derived from distinct gene classes that have been designated $\alpha, \mu, \pi$, etc. GSTs catalyze the conjugation of the sulfur atom of glutathione (GSH) to an electrophilic center of endogenous and exogenous compounds, thereby increasing their aqueous solubility for subsequent excretion. Being ubiquitous and quite abundant in mammalian tissues, they initiate the metabolism of a broad range of alkylating agents and therefore play a central role in the detoxification of many carcinogens as well as anticancer chemotherapeutic agents (Jakoby and Habig 1980; Mannervik 1985; Pickett and Lu 1989; Armstrong 1991, 1994, 1997; Hayes and Pulford 1995; Sheehan et al 2001; Dixon et al 2002). Of the superfamily, GST $\pi$ is especially important in cancer therapy because it is often expressed at significantly higher levels in preneoplastic and neoplastic cells (Sato et al 1984; Sugioka et al 1985; Suguoka et al 1985; Sato 1988, 1989; Muramatsu et al 1995). It has also been shown that elevated levels of total GST and overexpression of GST $\pi$ often accompany the development of drug resistance in tumors of patients undergoing chemotherapy (Morgan et al 1996; O’Brien and Tew 1996; Townsend and Tew 2003). Such factors have stimulated recent efforts to target GSTs as a primary objective in the discovery of anticancer agents (Flatgaard et al 1993; Lyttle et al 1994; Kauvar 1996; Rosario et al 2000; Townsend et al 2002).

We have been trying to turn the GST $\pi$-overexpression to the tumor's disadvantage by developing PABA/NO [ $O^{2}-\{2,4$-dinitro-5-[4-( $N$-methylamino)benzoyloxy]phenyl $\}$ 1-(N,N-dimethylamino)diazen-1-ium-1,2-diolate] that releases the established cytolytic 
agent nitric oxide (NO) upon metabolism by GST $\pi$ (Findlay et al 2004). PABA/NO belongs to a new family of anticancer prodrugs, the $O^{2}$-aryl diazeniumdiolates $\left(O^{2} \mathrm{ADs}\right)$, electrophilic species shown to transfer their aryl groups to attacking nucleophiles with cogeneration of ions that spontaneously release NO at physiological pH (Saavedra et al 2001). The GST-catalyzed GSH addition of PABA/NO proceeds with the formation of a Meisenheimer-complex intermediate (Figure 1a), and subsequently the leaving group of the reaction releases two moles of NO (Figure 1b). Therefore, within the GST-overexpressing cancer cells, the intracellular GSH is irreversibly consumed, and the NO thus generated could contribute to chemotherapy by inhibiting DNA synthesis, forming toxic reactive nitrogen/oxygen intermediates, and inhibiting enzymes capable of preventing or repairing cellular damage. PABA/NO produces antitumor effects comparable with cisplatin in a human ovarian cancer model grown in SCID mice and is also potent against proliferation of the OVCAR-3 cell line (Findlay et al 2004; Saavedra et al 2006). PABA/NO is GST $\pi$-selective, ie, it is more efficiently metabolized by GST $\pi$. It was designed on the basis of the mechanism of GSTcatalyzed reaction and the structures of GSTs, especially the structural information of GSTs at the transition state.

The attack of GSH on 1-chloro-2,4-dinitrobenzene (CDNB) is the basis for the most widely used spectrophotometric assay for measurement of GST activity (Habig et al 1974).<smiles>[X]c1cc([N+](=O)[O-])c([X])cc1[N+](=O)[O-]</smiles>

CDNB: $\mathrm{X}=\mathrm{H} ; \mathrm{Y}=\mathrm{Cl}$

PABA/NO: $X=0$<smiles>CNc1ccc(C(=O)O[Na])cc1</smiles>

(b)<smiles>CN(C)[N+]([O-])=N[O-]</smiles><smiles>O=[N+]([O-])c1cc([N+](=O)[O-])cc([N+](=O)[O-])c1</smiles><smiles>O=[N+]([O-])C1=CC([N+](=O)[O-])[C@H](Cl)C([N+](=O)[O-])=C1</smiles><smiles>CC(C)(C)C</smiles>
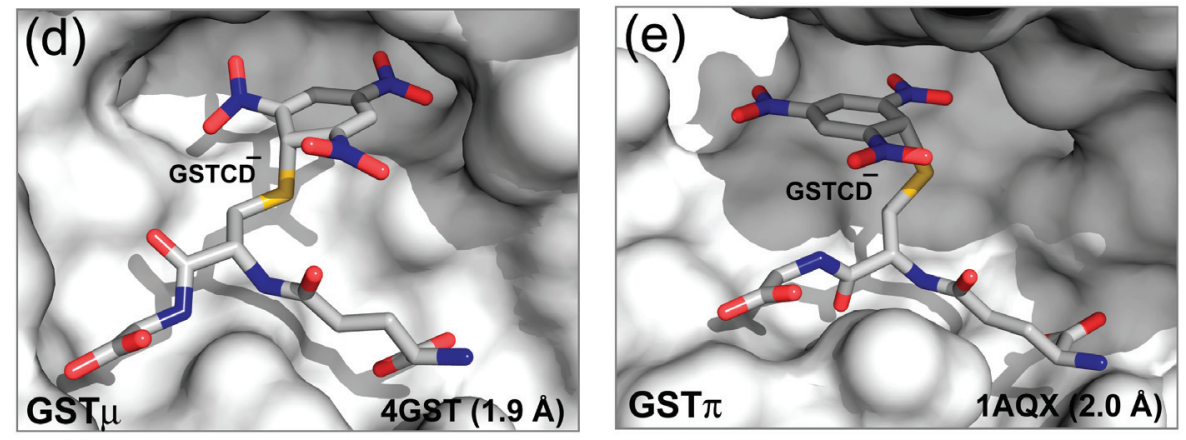

Figure I The Meisenheimer complex. (a) The mechanism of GST-catalyzed reaction of GSH with PABA/NO and with CDNB, showing the formation of the Meisenheimer complex as the reaction intermediate. (b) The diazeniumdiolate ion releases two moles of NO at neutral $\mathrm{pH}$. (c) A relatively stable model for the Meisenheimer complex is provided by the GSTCD ${ }^{-}$ion. (d) The GST $\mu \cdot \mathrm{GSTCD}^{-}$structure (PDB entry 4GST). (e) The GST $\pi \cdot \mathrm{GSTCD}^{-}$structure (PDB entry IAQX). In panels $d$ and e, the protein is shown as a molecular surface while the ligand as a stick model in atomic color scheme (carbon in gray, nitrogen in blue, oxygen in red, and sulfur in orange). 
As shown in Figure 1a, the reaction proceeds in solution via the formation of the Meisenheimer-complex intermediate (Miller 1968). Therefore, GST would stabilize the Meisenheimer-complex intermediate, at least to the extent that the intermediate resembles the transition state for its formation. However, the instability of the Meisenheimer complex has thus far precluded its structural elucidation. In contrast, the reversible reaction of GSH with 1,3,5-trinitrobenzene (TNB) to form the 1-(glutathion- $S$-yl)-2,4,6-trinitrocyclohexadienate anion (GSTCD-), a potent inhibitor of GST, provides a relatively stable model for this intermediate (Clark and Sinclair 1988; Graminski et al 1989) as shown in Figure 1c. To date, crystal structures of the GST $\mu \cdot \mathrm{GSTCD}^{-}$complex (PDB entry 4GST; Ji et al 1993) shown in Figure 1d and the GST $\pi \cdot G_{S T C D}-$ complex (PDB entry 1AQX; Prade et al 1997) shown in Figure 1e have been determined, which have guided us to model the GST $\alpha \cdot \mathrm{GSTCD}^{-}$complex. Together, the structures and the model played an essential role in our structure-based development of PABA/NO.

Structure-based drug design is a growing field in which remarkable advances have been made in recent years (Anderson 2003; Scapin 2006). Although the development of PABA/NO was briefly mentioned previously (Findlay et al 2004), the details, involving structural modification, molecular modeling, and enzymatic characterization of two key intermediate compounds, have not been reported. Besides, the structure of PABA/NO in the previous report was not correct (Saavedra et al 2006). Here, we present the structure-based design of PABA/NO in detail, during which the structures of different classes of GSTs, especially the structural information for the transition state of GSTcatalyzed reaction of GSH and CDNB, played an essential role. With this unique example, we demonstrate again the importance of structure-based approach in drug development and the value of structural information for the transition

state of an enzyme-driven reaction in structure-based drug design.

\section{Materials and methods Structural modifications of $O^{2} A D$ molecules}

Figure 2 depicts our structural modifications starting from GST $\alpha$-selective compounds 1 and resulting in GST $\pi$ selective compound 4 (PABA/NO). Compounds 1, 2, and 3 were prepared as previously described (Saavedra et al 2001), as was compound 4 (Findlay et al 2004).

\section{Modeling the GST $\alpha \cdot \mathrm{GSTCD}^{-}$complex}

Molecular modeling studies were carried out on an SGI Fuel workstation with program packages CNS and O (Jones and Kjeldgaard 1997; Brünger et al 1998). The initial model of GSTCD $^{-}$bound to GST $\alpha$ was built on the basis of the crystal structures of the GSTCD ${ }^{-}$found in the active sites of GST $\mu$ (PDB entry 4GST), and GST $\pi$ (PDB entry $1 \mathrm{AQX}$ ), and then it was docked into the active site of GST $\alpha$ in complex with the GSH adduct of ethacrynic acid (PDB entry 1GSE; Cameron et al 1995). The model complex, built in dimeric form because GSH interacts with the side chains from both subunits, was subject to geometry optimization using the conjugate gradient method by Powell embedded in CNS (Powell 1977; Brünger et al 1998). The Engh and Huber geometric parameters were used as the basis of the force field (Engh and Huber 1991). The coordinates of the GST $\alpha \cdot \mathrm{GSTCD}^{-}$model (Figure $3 \mathrm{a}$ ) are available from the corresponding author upon request.

\section{Modeling GST with bound Meisenheimer complex of $O^{2} A D$}

The initial models of the Meisenheimer complex of compounds 1 and $4\left(\mathrm{GS1}^{-}\right.$and $\mathrm{GS}^{-}$) bound to the three GST isozymes were

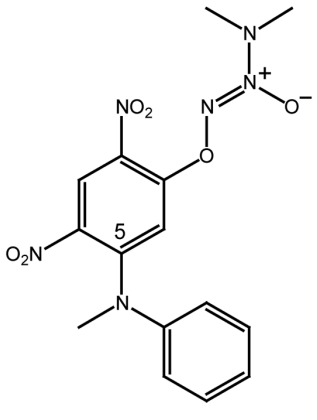

3

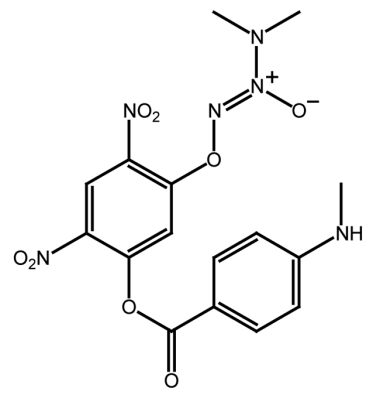

4 (PABA/NO)

Figure 2 Structure-based design of PABA/NO. Structural modifications of the GST $\alpha$-selective compounds I and 2 have led to the GST $\pi$-selective compound 4 (PABA/NO). 

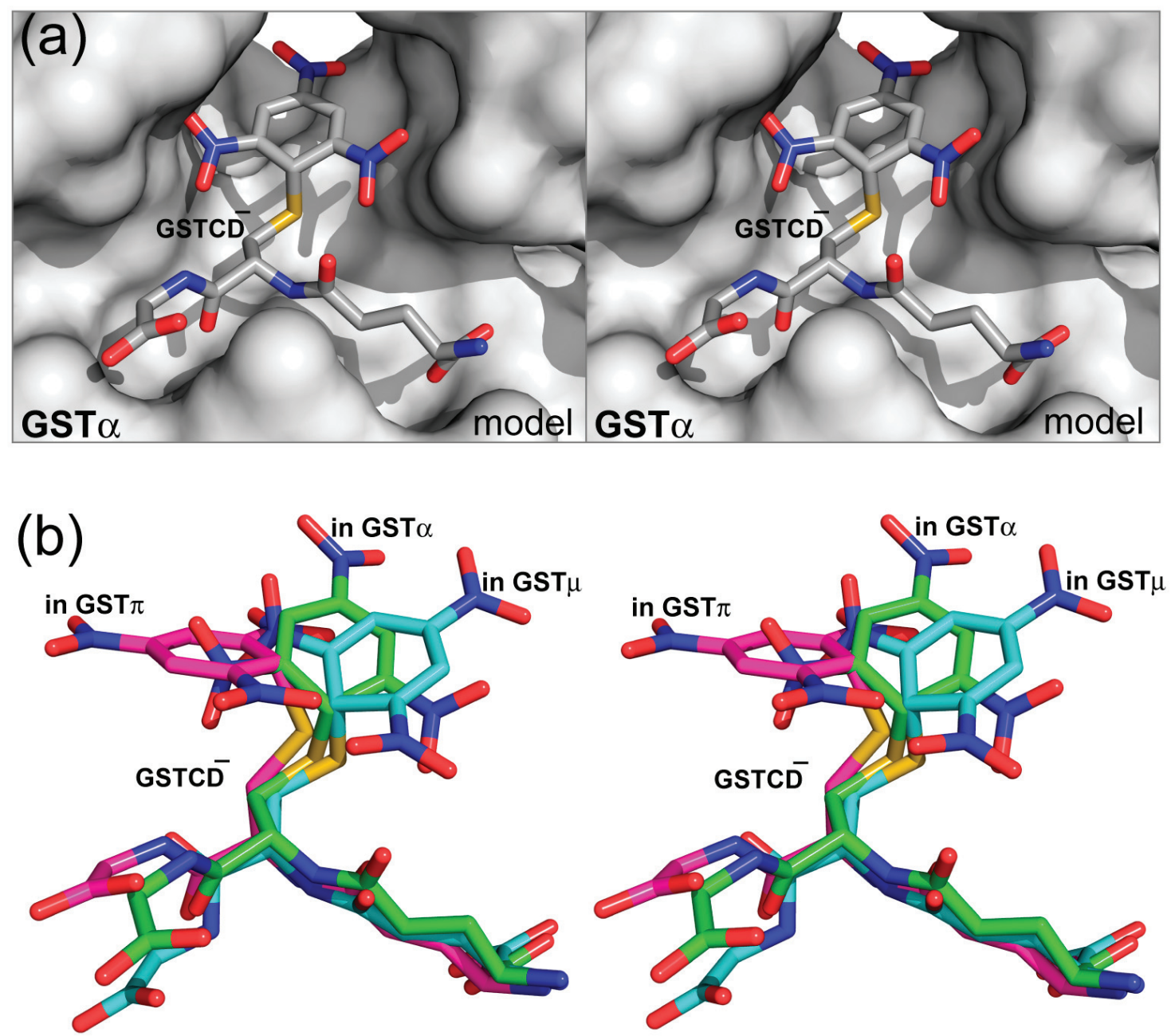

Figure 3 The GST $\alpha \cdot G S T C D^{-}$model. (a) Stereoview showing the GST $\alpha \cdot G S T C D^{-}$model, constructed on the basis of the crystal structure of GST $\alpha$ in complex with the GSH conjugate of ethacrynic acid (PDB entry IGSE), the GST $\mu \cdot G_{S T C D}^{-}$structure (PDB entry 4GST), and the GST $\pi \cdot G^{-} S_{C D}^{-}$structure (PDB entry IAQX). The protein is shown as a molecular surface while the ligand as a stick model in atomic color scheme (carbon in gray, nitrogen in blue, oxygen in red, and sulfur in orange). (b) Stereoview showing the alignment of the GSTCD- ions in the GST $\bullet^{\bullet} \mathrm{GSTCD}^{-}$model, in the GST $\mu$ GSTCD$^{-}$structure (PDB entry 4GST), and in the GST $\pi \bullet^{\circ G S T C D}{ }^{-}$structure (PDB entry IAQX). The GSTCD- ion is shown as a stick model in atomic color scheme (nitrogen in blue, oxygen in red, and sulfur in orange; carbon in green when bound in GST $\alpha$, cyan in GST $\mu$, and magenta in GST $\pi$ ).

built on the basis of the crystal structures of compounds $\mathbf{1}$ and $\mathbf{4}$ (Saavedra et al 2001, 2006) and the GSTCD- structures in the GST $\alpha \cdot \mathrm{GSTCD}^{-}$model, the GST $\mu \cdot \mathrm{GSTCD}^{-}$structure (PDB entry $4 \mathrm{GST}$ ), and the GST $\pi \cdot \mathrm{GSTCD}^{-}$structure (PDB entry 1AQX). The initial models of GS2- ${ }^{-}$were derived from those of GS4- by removing the 5-position bulky group, and then they were docked into the active sites of the corresponding GSTs. The dimeric model complexes, except for GST $\pi \cdot \mathrm{GS1}^{-}$(Figure 4c) and GST $\propto \cdot \mathrm{GS4}^{-}$(Figure $4 \mathrm{~g}$ ), were subject to geometry optimization in the same manner as for the GST $\bullet^{\cdot G S T C D}{ }^{-}$complex.

\section{Kinetic studies}

The recombinant GST isoenzymes were either purchased from Panvera or purified as described previously (Zimniak et al 1994). The reaction mixture, in a final volume of $1 \mathrm{~mL}$, contained $0.1 \mathrm{M}$ phosphate buffer ( $\mathrm{pH}$ 6.5-7.0), 1-5 $\mu \mathrm{g} / \mathrm{mL}$ of the desired GST protein, 1-2 mM GSH and varying concentrations of compound 1 or compound $2(20-200 \mu \mathrm{M})$. Change in absorbance was monitored spectrophotometrically at $380 \mathrm{~nm}$. The enzyme activity was calculated using an extinction coefficient of $3.58 \mathrm{mM}^{-1} \mathrm{~cm}^{-1}$. Kinetic parameters were calculated using the Michaelis-Menten equation by software KiNET (Kinexus, Vancouver, Canada). The experiments were repeated three times and the results were averaged.

The same set of kinetic studies was carried out in two independent laboratory settings and the results were consistent.

\section{Illustrations}

The schematic illustrations for the crystal structures and the models were generated with PyMOL (DeLano 2002). 


\section{Results and discussion}

\section{Structure of a transition-state analog in complex with GST $\alpha$}

GST $\alpha$ is the predominant isoform of GST in human liver, whereas GST $\pi$ is most abundant in extrahepatic tissues and is overexpressed in many types of tumors. In order to target tumor cells while avoiding toxicity to the liver, structural information is needed for GST $\alpha$ in complex with the transition-state analog $\mathrm{GSTCD}^{-}$. On the basis of available structural information, we have built a model of the GST $\alpha \cdot \mathrm{GSTCD}^{-}$complex (Figure 3a). Together, the GST $\mu \cdot \mathrm{GSTCD}^{-}$structure (PDB entry 4GST), the GST $\pi \cdot \mathrm{GSTCD}^{-}$structure (PDB entry 1AQX), and the GST $\alpha \cdot \mathrm{GSTCD}^{-}$model have shown that the conformation of the $\mathrm{GSTCD}^{-}$ion exhibits such dramatic differences when it is bound in the active site of different GSTs that the aromatic ring of the GSTCD- ion stretches out in distinct directions (Figure 3b), providing the guidance for our structure-based modification of $O^{2} \mathrm{AD}$ molecules toward the GST $\pi$-selective PABA/NO.

\section{Compound I is GST $\alpha$-selective}

Compound 1 (Figure 2) is the first $O^{2} \mathrm{AD}$ molecule we have ever synthesized (Saavedra et al 2001). All three classes of GSTs catalyze the NO release of $\mathbf{1}$. However, it is GST $\alpha$-selective, 2-fold over GST $\mu$ and 145-fold over GST $\pi$ (Table 1). To understand the structural basis for the different catalytic efficiencies, we have constructed model complexes of the three GSTs with GS1 ${ }^{-}$, the Meisenheimer complex of 1 . The models of GST $\alpha \cdot \mathrm{GS}^{-}$(Figure 4a) and GST $\mu \cdot \mathrm{GS1}^{-}$(Figure 4b) suggest favorable interactions between the protein and the reaction intermediate. The GST $\pi \cdot \mathrm{GS1}^{-}$model, however, suggests unfavorable interaction between the diethyl group of the diazeniumdiolate moiety and the protein (Figure 4c). Therefore, the first modification of $\mathbf{1}$ was to replace the diethylamino group of the diazeniumdiolate moiety with a dimethylamino group, which was predicted to improve the fit of the reaction intermediate derived from the resulting compound to GST $\pi$.

\section{Compound 2 indicates that our design is on the right track}

As expected, compound 2 (Figure 2) is indeed a better substrate for GST $\pi$ than compound 1 . The catalytic efficiency of GST $\pi$ toward 2 exhibits a noticeable increase (Table 2), which is likely due to the reduced steric interactions between the diazeniumdiolate moiety of the reaction intermediate and the protein (Figure 4c). Moreover, the two other GSTs are less active toward 2, with a 4-fold reduction in the catalytic efficiency for GST $\alpha$ and a 3-fold reduction for GST $\mu$ (Table 2). Although it is not as obvious, the reduction of the hydrophobic interactions between the protein and the diazeniumdiolate moiety of GS2- ${ }^{-}$, the Meisenheimer complex of 2 , may have played a role. Although 2 is still GST $\alpha$-selective, 1.5-fold over GST $\mu$ and 24-fold over GST $\pi$, the improvement has indicated that our structure-based design is on the right track. The next modification is to further reduce the GST $\alpha$ selectivity while increasing the GST $\pi$ selectively.

We have constructed model complexes of $\mathrm{GS2}^{-}$with GST $\alpha$ (Figure 4d), GST $\mu$ (Figure 4e), and GST $\pi$ (Figure 4f). The models suggest that the addition of a sterically demanding constituent at position 5 of the aryl ring should increase its accommodation in the active site of GST $\pi$ (Figure 4f) while decreasing its accommodation in the active site of GST $\alpha$ (Figure 4d). Thus, compound 3 was designed and synthesized (Figure 2). Unfortunately, the solubility of $\mathbf{3}$ was too low, rendering its enzymatic characterization impossible. Further modification aimed to improve the solubility has led to the synthesis of compound 4 , ie, the PABA/NO (Figure 2).

\section{PABA/NO (Compound 4) is GST $\pi$-selective}

As expected, PABA/NO (compound 4) is a better substrate for GST $\pi$ compared to GST $\alpha$ (Findlay et al 2004). The model of GST $\alpha$ in complex with the Meisenheimer complex of 4 $\left(\mathrm{GST} \alpha \cdot \mathrm{GS}^{-}\right)$suggests unfavorable interaction between the bulky group at position 5 of the aryl ring system (Figure $4 \mathrm{~g}$ ). In contrast, both GST $\mu$ and GST $\pi$ can accommodate this

Table I Kinetic data for human GST isoenzymes with Compound I as the variable substrate

\begin{tabular}{lllll}
\hline Enzyme $^{\mathrm{a}}$ & $\mathbf{K}_{\mathbf{m}}(\mu \mathrm{M})$ & $\mathbf{V}_{\max }\left(\mathbf{n m o l ~} \mathbf{m i n}^{-1} \mathbf{m g}^{-1}\right)$ & $\mathbf{k}_{\mathrm{cat}}\left(\mathbf{s}^{-1}\right)$ & $\mathbf{k}_{\mathrm{cat}} / \mathbf{K}_{\mathbf{m}}{ }^{\mathrm{b}}\left(\mathbf{m M M}^{-1} \mathbf{s}^{-1}\right)$ \\
\hline GST $\alpha$ & $196 \pm 63$ & $200898 \pm 37633$ & 171 & 872 \\
GST $\mu$ & $171 \pm 51$ & $85508 \pm 14322$ & 73 & 427 \\
GST $\pi$ & $523 \pm 143$ & $4017 \pm 848$ & 3.1 & 6 \\
\hline
\end{tabular}

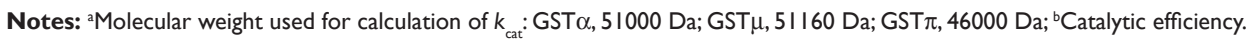


Table 2 Kinetic data for human GST isoenzymes with Compound $\mathbf{2}$ as the variable substrate

\begin{tabular}{lllll}
\hline Enzyme $^{\mathbf{a}}$ & $\mathbf{K}_{\mathrm{m}}(\mu \mathbf{M})$ & $\mathbf{V}_{\max }\left(\mathbf{n m o l ~} \mathbf{m i n}^{-1} \mathbf{m g}^{-1}\right)$ & $\mathbf{k}_{\mathrm{cat}}\left(\mathbf{s}^{-1}\right)$ & $\mathbf{k}_{\mathrm{cat}} / \mathbf{K}_{\mathrm{m}}{ }^{\mathrm{b}}\left(\mathbf{m M M}^{-1} \mathbf{s}^{-1}\right)$ \\
\hline GST $\alpha$ & $136 \pm 27$ & $37932 \pm 3793$ & 32 & 237 \\
GST $\mu$ & $1910 \pm 327$ & $337169 \pm 53003$ & 287 & 150 \\
GST $\pi$ & $237 \pm 404$ & $2997 \pm 404$ & 2.2 & 9.7
\end{tabular}

Notes: ${ }^{a}$ Molecular weight used for calculation of $k_{\text {cat }}$ : GST $\alpha, 51000 \mathrm{Da}$; GST $\mu, 51160 \mathrm{Da}$; GST $\pi, 46000 \mathrm{Da}$; ${ }^{\mathrm{b} C a t a l y t i c}$ efficiency.

group (Figure 4, panels $\mathrm{h}$ and i). Thus, the structure-based modifications of 1 have reversed the GST isoenzyme selectively of $O^{2} \mathrm{AD}$.

\section{GST $\mu$ activity toward $O^{2} A D$}

The expression of GST $\mu$ is null in $50 \%$ of the human population due to a gene deletion (Rowe et al 1997). Therefore, our current effort has been focused on GST $\alpha$ and GST $\pi$ while keeping GST $\mu$ in the scope of our investigation for better understanding the reaction mechanism. Strategies have been developed to avoid GST $\mu$ for the development of next-generation $O^{2} \mathrm{AD}$ anticancer prodrugs.

\section{The Meisenheimer complex of $O^{2} A D$}

We have previously shown that the transient Meisenheimer complex is generated in the reaction of compound 1 (Saavedra et al 2001). On the basis of this mechanism, during which the Meisenheimer complex of $O^{2} \mathrm{AD}$ is formed (Figure 1a), we have used the GST $\mu \cdot \mathrm{GSTCD}^{-}$structure (Figure 1d), the GST $\pi \cdot \mathrm{GSTCD}^{-}$structure (Figure 1e), and the GST $\alpha \cdot \mathrm{GSTCD}^{-}$model (Figure $3 \mathrm{a}$ ) to guide the construction of the three GSTs in complex with the Meisenheimer complex of $O^{2} \mathrm{ADs}$ (Figure 4).

The model complexes of GST with the Meisenheimer complexes of $O^{2} \mathrm{AD}$ have played a crucial role in guiding
1
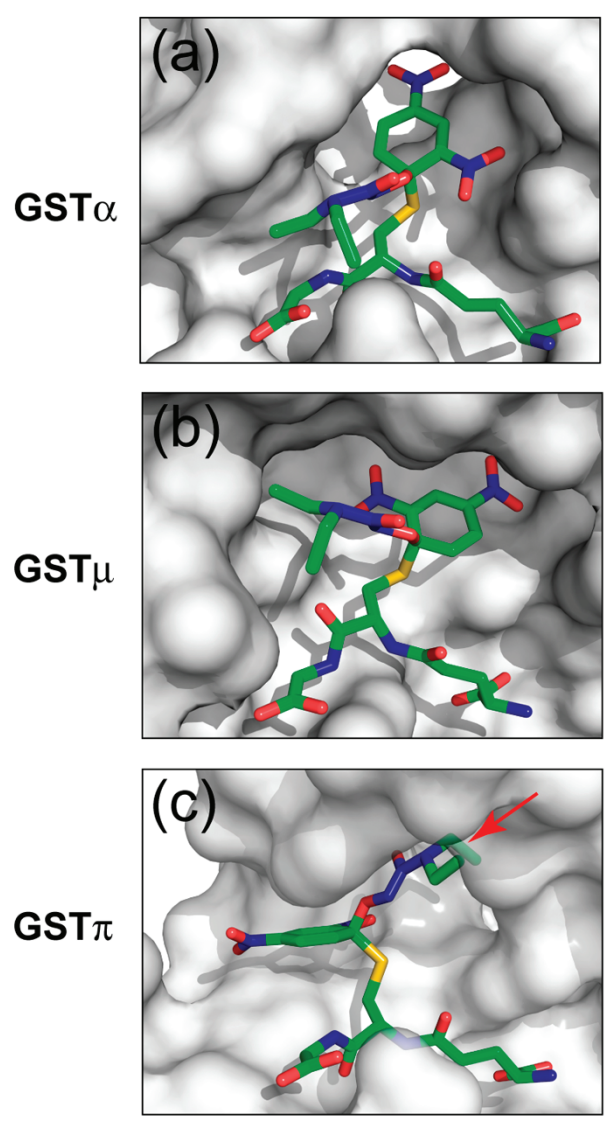

2
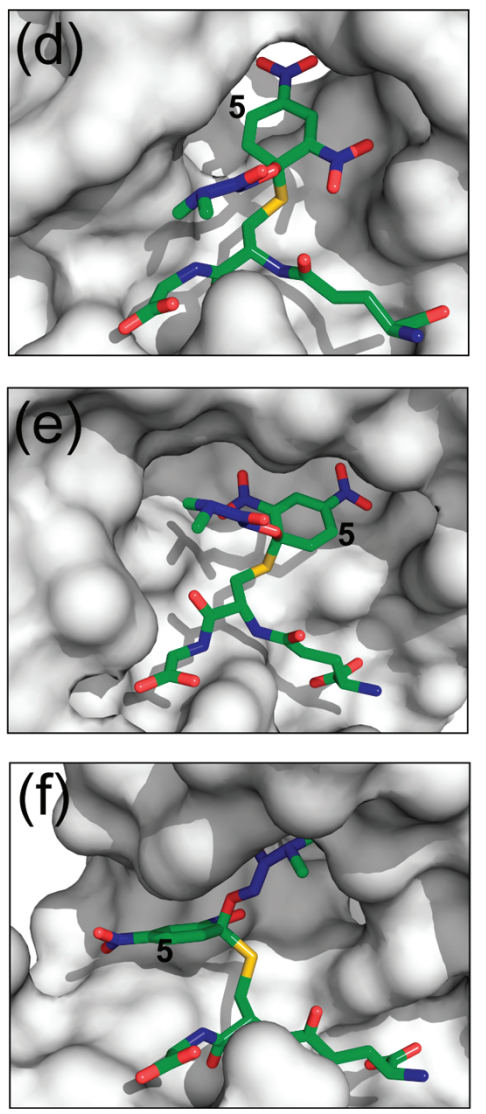

4 (PABA/NO)
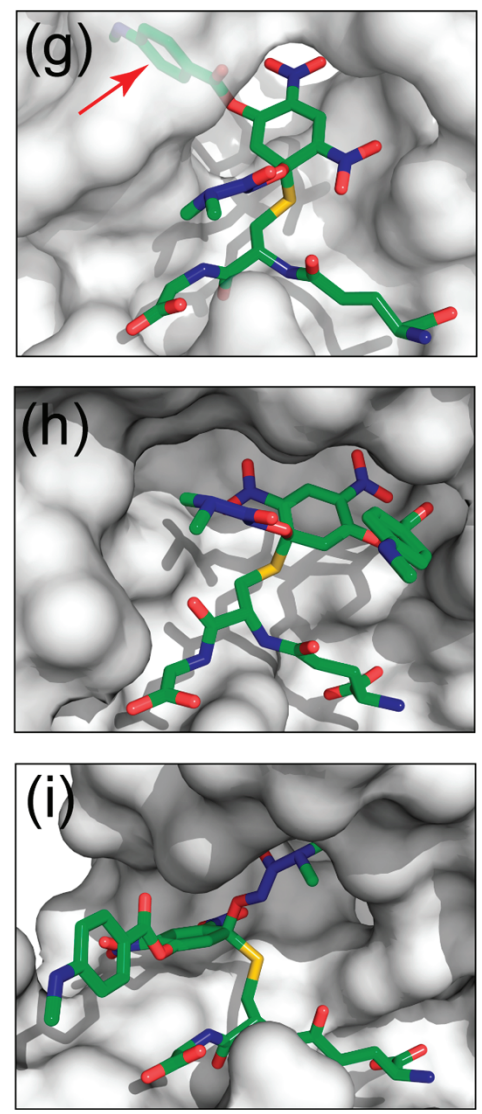

Figure 4 Models of the three GST isozymes with bound Meisenheimer complex of compound I, 2, and 4. The GST active sites are illustrated as non-transparent surfaces, except for a transparent surface in panels $\mathrm{c}$ and $\mathrm{g}$ to show the unfavorable interaction between the ligand and the protein (indicated with red arrows), and the ligands as stick models in atomic color scheme (carbon in green, nitrogen in blue, oxygen in red, and sulfur in orange). 
the modification of compounds $\mathbf{1}$ and $\mathbf{2}$, resulting in the development of compound 4, the GST $\pi$-selective PABA/ $\mathrm{NO}$ as an anticancer drug lead. The key factors in this design include (1) understanding the difference in the shape and property of the substrate-binding site of different GSTs and (2) using the structures of GSTCD ${ }^{-}$in different GST complexes to guide the molecular modeling. For example, the substrate-binding site is broad in both GST $\mu$ and GST $\pi$ (Figure1 panels d and e). However, it is virtually a hydrophobic cavity in GST $\mu$, but is approximately half hydrophobic and half hydrophilic in GST $\pi$ ( $\mathrm{Ji}$ et al 1997). It is mainly the property of the binding site that dictates the orientation of the trinitrophenyl ring system of $\mathrm{GSTCD}^{-}$in these two isoenzymes. In support of this notion, the ring system of the GSH adduct of phenanthrene 9,10-oxide also points into opposite directions in these two isoenzymes ( $\mathrm{Ji}$ et al 1994, 1997). Unlike GST $\mu$ and GST $\pi$, GST $\alpha$ has a narrower substrate-binding site, which limits the conformational freedom of the trinitrophenyl moiety of $\mathrm{GSTCD}^{-}$(Figure 3a).

\section{Conclusions}

This paper describes the details on structural modification, molecular modeling, and enzymatic characterization for structure-based design of PABA/NO. The design was efficient because it was on the basis of the reaction mechanism and the structures of related GST isozymes at both the ground state and the transition state. The groundstate structures outlined the shape and property of the substrate-binding site in different isozymes, and the known transition-state structures helped us build a model of GST $\alpha$ at the transition state of which the structural information was not available. The structural information thus derived for the transition state, showing distinct conformations of the Meisenheimer complex of $O^{2} \mathrm{ADs}$ in the active site of different isozymes, guided the modifications of the molecular structure of $O^{2} \mathrm{AD}$ molecules. Two key alterations of a GST $\alpha$-selective compound, the reduction of the size of the amino group and the introduction of the bulky group, led to the GST $\pi$-selective PABA/NO.

\section{Acknowledgments}

This research was supported by the Intramural Research Program of the NIH, National Cancer Institute (NCI), Center for Cancer Research, and by federal funds from the NCI under contract N01-CO-12400. The content of this publication does not reflect the views or policies of the Department of Health and Human Services, nor does mention of trade names, commercial products, or organizations imply endorsement by the US Government.

\section{References}

Anderson AC. 2003. The process of structure-based drug design. Chem Biol, 10:787-97.

Armstrong RN. 1991. Glutathione S-transferases: reaction mechanism, structure, and function. Chem Res Toxicol, 4:131-40.

Armstrong RN. 1994. Glutathione S-transferases: structure and mechanism of an archetypical detoxication enzyme. Adv Enzymol Relat Areas Mol Biol, 69:1-44.

Armstrong RN. 1997. Structure, catalytic mechanism, and evolution of the glutathione transferases. Chem Res Toxicol, 10:2-18.

Brünger AT, Adams PD, Clore GM, et al. 1998. Crystallography and NMR system: A new software suite for macromolecular structure determination. Acta Crystallogr D, 54:905-21.

Cameron AD, Sinning I, L'Hermite G, et al. 1995. Structural analysis of human alpha-class glutathione transferase A1-1 in the apo-form and in complexes with ethacrynic acid and its glutathione conjugate. Structure, 3:717-27.

Clark AG, Sinclair M. 1988. The Meisenheimer complex of glutathione and trinitrobenzene. A potent inhibitor of the glutathione S-transferase from Galleria mellonella. Biochem Pharmacol, 37:259-63.

DeLano WL. 2002. The PyMOL Molecular Graphics System. San Carlos, CA: Delano Scientific.

Dixon DP, Lapthorn A, Edwards R. 2002. Plant glutathione transferases. Genome Biol, 3:REVIEWS3004.

Engh RA, Huber R. 1991. Accurate bond and angle parameters for X-ray protein structure refinement. Acta Crystallogr A, 47:392-400.

Findlay VJ, Townsend DM, Saavedra JE, et al. 2004. Tumor cell responses to a novel glutathione S-transferase-activated nitric oxide-releasing prodrug. Mol Pharmacol, 65:1070-9.

Flatgaard JE, Bauer KE, Kauvar LM. 1993. Isozyme specificity of novel glutathione-S-transferase inhibitors. Cancer Chemother Pharmacol, 33:63-70.

Graminski GF, Zhang PH, Sesay MA, et al. 1989. Formation of the 1-(S-glutathionyl)-2,4,6-trinitrocyclohexadienate anion at the active site of glutathione S-transferase: evidence for enzymic stabilization of sigma-complex intermediates in nucleophilic aromatic substitution reactions. Biochemistry, 28:6252-8.

Habig WH, Pabst MJ, Jakoby WB. 1974. Glutathione S-transferases. The first enzymatic step in mercapturic acid formation. $J$ Biol Chem, 249:7130-9.

Hayes JD, Pulford DJ. 1995. The glutathione S-transferase supergene family: regulation of GST and the contribution of the isoenzymes to cancer chemoprotection and drug resistance. Crit Rev Biochem Mol Biol, 30:445-600.

Jakoby WG, Habig WH. 1980. Enzymatic Basis of Detoxication. New York: Academic Press.

Ji X, Armstrong RN, Gilliland GL. 1993. Snapshots along the reaction coordinate of an $\mathrm{S}_{\mathrm{N}} \mathrm{Ar}$ reaction catalyzed by glutathione transferase. Biochemistry, 32:12949-54.

Ji X, Johnson WW, Sesay MA, et al. 1994. Structure and function of the xenobiotic substrate-binding site of a glutathione S-transferase as revealed by X-ray crystallographic analysis of product complexes with the diastereomers of 9-(S-glutathionyl)-10-hydroxy-9,10-dihydrophenanthrene. Biochemistry, 33:1043-52.

Ji X, Tordova M, O’Donnell R, et al. 1997. Structure and function of the xenobiotic substrate-binding site and location of a potential non-substrate-binding site in a class pi glutathione S-transferase. Biochemistry, 36:9690-702.

Jones TA, Kjeldgaard M. 1997. Electron-density map interpretation. Methods Enzymol, 277:173-208.

Kauvar LM. 1996. Peptide mimetic drugs: a comment on progress and prospects. Nat Biotechnol, 14:709. 
Lyttle MH, Hocker MD, Hui HC, et al. 1994. Isozyme-specific glutathione-S-transferase inhibitors: design and synthesis. $J$ Med Chem, 37:189-94.

Mannervik B. 1985. The isoenzymes of glutathione transferase. Adv Enzymol Relat Areas Mol Biol, 57:357-417.

Miller J. 1968. Reaction Mechanisms in Organic Chemistry. New York: Elsevier.

Morgan AS, Ciaccio PJ, Tew KD, et al. 1996. Isozyme-specific glutathione S-transferase inhibitors potentiate drug sensitivity in cultured human tumor cell lines. Cancer Chemother Pharmacol, 37:363-70.

Muramatsu M, Suzuki T, Hisatake K, et al. 1995. Regulation of glutathione transferase P-expression during hepatocarcinogenesis of the Rat. In: Vermeulen NPE, Mulder GJ, Nieuwenhuyse H, et al. (eds).Glutathione S-Transferease - Structure, Function and Clinical Implications. London: Taylor and Francis. pp. 85-95.

O'Brien ML, Tew KD. 1996. Glutathione and related enzymes in multidrug resistance. Eur J Cancer, 32A:967-78.

Pickett CB, Lu AY. 1989. Glutathione S-transferases: gene structure, regulation, and biological function. Annu Rev Biochem, 58:743-64.

Powell MJD. 1977. Restart procedures for the conjugate gradient method. Math Prog, 12:241-54.

Prade L, Huber R, Manoharan TH, et al. 1997. Structures of class pi glutathione S-transferase from human placenta in complex with substrate, transition-state analogue and inhibitor. Structure, 5:1287-95.

Rosario LA, O'Brien ML, Henderson CJ, et al. 2000. Cellular response to a glutathione S-transferase P1-1 activated prodrug. Mol Pharmacol, 58:167-74.

Rowe JD, Nieves E, Listowsky I. 1997. Subunit diversity and tissue distribution of human glutathione S-transferases: interpretations based on electrospray ionization-MS and peptide sequence-specific antisera. Biochem J, 325(Part 2):481-6.

Saavedra JE, Srinivasan A, Bonifant CL, et al. 2001. The secondary amine/ nitric oxide complex ion $\mathrm{R}_{2} \mathrm{~N}\left[\mathrm{~N}(\mathrm{O}) \mathrm{NO}^{-}\right.$as nucleophile and leaving group in $\mathrm{S}_{\mathrm{N}} \mathrm{Ar}$ reactions. J Org Chem, 66:3090-8.
Saavedra JE, Srinivasan A, Buzard GS, et al. 2006. PABA/NO as an anticancer lead: analogue synthesis, structure revision, solution chemistry, reactivity toward glutathione, and in vitro activity. $J$ Med Chem, 49:1157-64.

Sato K. 1988. Glutathione S-transferases and hepatocarcinogenesis. Jpn J Cancer Res, 79:556-72.

Sato K. 1989. Glutathione transferases as markers of preneoplasia and neoplasia. Adv Cancer Res, 52:205-55.

Sato K, Kitahara A, Satoh K, et al. 1984. The placental form of glutathione S-transferase as a new marker protein for preneoplasia in rat chemical hepatocarcinogenesis. Jpn J Cancer Res, 75:199-202.

Scapin G. 2006. Structural biology and drug discovery. Curr Pharm Des, 12:2087-97.

Sheehan D, Meade G, Foley VM, et al. 2001. Structure, function and evolution of glutathione transferases: implications for classification of non-mammalian members of an ancient enzyme superfamily. Biochem J, 360:1-16.

Sugioka Y, Fujii-Kuriyama Y, Kitagawa T, et al. 1985. Changes in polypeptide pattern of rat liver cells during chemical hepatocarcinogenesis. Cancer Res, 45:365-78.

Suguoka Y, Kano T, Okuda A, et al. 1985. Cloning and the nucleotide sequence of rat glutathione S-transferase P cDNA. Nucleic Acids Res, 13:6049-57.

Townsend D, Tew K. 2003. Cancer drugs, genetic variation and the glutathione-S-transferase gene family. Am J Pharmacogenomics, 3:157-72.

Townsend DM, Shen H, Staros AL, et al. 2002. Efficacy of a glutathione S-transferase pi-activated prodrug in platinum-resistant ovarian cancer cells. Mol Cancer Ther, 1:1089-95.

Zimniak P, Nanduri B, Pikula S, et al. 1994. Naturally occurring human glutathione S-transferase GSTP1-1 isoforms with isoleucine and valine in position 104 differ in enzymic properties. Eur J Biochem, 224:893-9. 